\title{
Integración de conocimiento en restaurantes mediante el análisis multicriterio para la toma de decisiones
}

\author{
Volumen XIX No (2). Julio-Diciembre 2019. Pág. 25-38
}

ISSN: 0121-1048 IMPRESO ISSN: 2422-3220 EN LÍNEA

\author{
León Santiesteban, Martín• \\ Universidad Autónoma de Occidente, Departamento \\ de Ciencias Económico Administrativa. Blvd. Lola \\ Beltrán. Km. 1.5, Culiacán, Sinaloa. \\ martin.leon@udo.mx
}

\author{
Larrañaga Núñez, Ana María \\ Universidad Autónoma de Occidente, Departamento \\ de Ciencias Económico Administrativa. Blvd. Lola \\ Beltrán. Km. 1.5, Culiacán, Sinaloa, México. \\ Amlarranaga2607@gmail.com
}

\section{Resumen}

El conocimiento es un bien intangible de la organización que genera innovación, ventaja competitiva y eficacia, por lo que es necesaria su gestión permanente y medición apoyada con herramientas como el análisis multicriterio para la toma de decisiones. En las empresas restauranteras ubicadas en el destino turístico de Mazatlán, Sinaloa, México, se realizan actividades de innovación que integran conocimiento por fuentes de libre acceso, compra y cooperación y que incluyen una serie de categorías, por lo que el objetivo es identificar la alternativa de decisión para la integración de conocimiento mediante un método multiatributo en la innovación de procesos. El método utilizado es el enfoque de ponderación aditiva simple que a través del estudio de caso llevó a la selección, al cálculo de la matriz de desempeño y al ranking de alternativas. En los hallazgos se realizó un ordenamiento y destacan tres restaurantes que mejor lograron la integración de conocimiento y tecnología del resultado global y en lo individual la tendencia al uso de fuentes de libre acceso. En conclusión, al ser difícil de imitar la innovación de procesos es relevante estudiar las alternativas de decisión de conocimiento e identificar la gestión de este recurso intangible en las empresas para diferenciarse en un mercado en crecimiento.

Palabras clave: Conocimiento, gestión, análisis multicriterio para la toma de decisiones, restaurante

Códigos JEL: I210, M100, C6, Z300

·Citar: León Santiesteban, Martín y Larrañaga Núñez, Ana María (2019). Integración de conocimiento en restaurantes mediante el análisis multicriterio para la toma de decisiones. Inquietud Empresarial, 19(2), 25-38. 


\title{
Integration of knowledge in restaurants through multicriteria analysis for decision making
}

\begin{abstract}
Knowledge is an intangible good of the organization that generates innovation, competitive advantage and efficiency, which is why necessary its permanent management and measurement supported with tools such as multicriteria analysis for decision making. In the restaurant companies located in the tourist destination of Mazatlan, Sinaloa, Mexico, innovation activities were carried out that integrate knowledge by free access sources, purchase and cooperation and that include a series of categories, so the objective is to identify the decision alternative for the integration of knowledge through a multi-attribute method in process innovation. The method used is the simple additive weighting approach, which through the case study led to the selection of alternatives, to the calculus of the performance matrix and the ranking of alternatives. In the findings, an order was made, and three restaurants stand out that better achieved the integration of knowledge and technology of the global result and in the individual the tendency to use sources of free access. In conclusion, since it is difficult to imitate process innovation, it is relevant to study the knowledge decision alternatives and identify the management of this intangible resource in companies to differentiate in a growing market.
\end{abstract}

Keywords: Knowledge, management, multicriteria analysis for decision making, restaurant.

JEL Codes: I210, M100, C6, Z300

\section{INTRODUCCIÓN}

El conocimiento en la organización es un bien intangible, que se caracteriza por su renovación y que adquiere valor para la economía al compartirse con otras personas internamente, pero también con suministradores o clientes (Hernández, 2016). Además, este recurso es la base de aprendizajes e innovaciones por lo que resulta una estrategia de gestión que entre otras “disminuye la redundancia de tareas y el número de errores en su ejecución, al aprovechar la experiencia existente dentro de la organización" (Medina, Medina \& Nogueira, 2017, p. 17). De esta manera, las organizaciones con orientación al aprendizaje crean un conocimiento aplicable que puede reducir la incertidumbre en el medio ambiente a través de la toma de decisiones (Fraj, Matute \& Melero, 2015).
El sistema turístico integra al sector de alimentos y bebidas como una actividad que deja amplios ingresos económicos (Lee, Hallak \& Sardeshmukh, 2016; Pérez \& Alonso, 2017) además de ser un mercado altamente competitivo por el número de empresarios que en él invierten (Aparecido \& Marques, 2016), lo que es motivado por el espacio geográfico donde se desarrolla y por el tamaño de la empresa. Estas organizaciones en su cotidianidad se mantienen ocupadas, lo que les dificulta identificar el conocimiento disponible o acumulado ya sea organizacionalmente, en gerentes o empleados para una adecuada planificación. (Zaei \& Zaei, 2014).

Por lo anterior, para asegurar el desempeño eficiente, innovar y generar ventajas competitivas de estas organizaciones se requiere de procesos y estrategias que acorde al desarrollo de las tecnologías de la 
información y la comunicación conlleven al aceleramiento de los flujos de conocimientos tácitos y explícitos lo que demanda una gestión del conocimiento y una toma de decisiones eficaz (Franch, Herrera \& Lozada, 2013). Los resultados que se derivan de estas actividades aportan un valor agregado en los procesos del sector productivo, dando como resultado mayor competitividad $y$ crecimiento económico en las empresas (Salas \& Garza, 2017).

En este sentido, el conocimiento es una fuente de la innovación, la que debe gestionarse para su apropiación y manejo, así como buscar la forma de colocarlo en común en la organización. En esta gestión se deben identificar los vínculos externos y los flujos de conocimiento al interior de la empresa y establecer relaciones estrechas con otras empresas, clientes o con instituciones de investigación. (Organización para la Cooperación \& Desarrollo Económico [OCDE], 2006).

$\mathrm{Al}$ mismo tiempo, al considerarse la toma de decisiones como una de las principales actividades del gestor en la organización que involucra la evaluación de opciones y decidirse por alguna alternativa que mejor se ajuste a los objetivos de la empresa (Flores \& Leyva, 2015), el análisis multicriterio para la toma de decisiones (MCDA) por sus siglas en inglés, coadyuva con el decisor en ambientes y problemas complejos indeterminados, con diversos determinantes y alternativas (Leyva, Avilés \& Zepeda, 2009).

En este escenario, la integración de conocimiento y MCDA en la empresa turística conlleva a la innovación de procesos. Esta perspectiva acorde a lo que mencionan Barbosa y Dominique (2012) la innovación coadyuva con las organizaciones y las empresas en la construcción de las bases presentes para el desarrollo futuro, por lo que debido al incremento de la globalización y la desregulación la innovación de productos pasa a un segundo plano al imitarse fácilmente con lo que se privilegia la innovación de procesos Weiermair (2004). Además, el estudio del conocimiento y el análisis multicriterio para la toma de decisiones por sus contribuciones a la organización se considera relevante, y son escasas las investigaciones publicadas que incorporen estas categorías con estudios del turismo (Mardani et al. 2015).

Por lo anterior, el objetivo de este artículo es identificar la alternativa de decisión para la integración de conocimiento a través de un método multiatributo, en la innovación de proceso en la empresa restaurantera ubicada en el destino turístico de Mazatlán, Sinaloa, México.

Para el caso de la empresa mexicana, Rodríguez (2004) señala que en la toma de decisiones los aspectos considerados son los valores, las creencias, la intención y la buena ventaja competitivas; en segundo término, la actividad turística del destino y con ello en su competitividad al contar con una oferta de servicios restauranteros diferenciada.

La industria restaurantera se define como los servicios de preparación de alimentos y bebidas para su consumo inmediato en el mismo establecimiento o fuera de éste. (Instituto Nacional de Estadística y Geografía [INEGI], 2016a). Al respecto el instituto menciona que los establecimientos dedicados a la preparación de alimentos y bebidas en el 2013 generaron 177 mil 145 millones de pesos, esto representó el 1.1\% del PIB total en México. Aunado a estos indicadores en el destino turístico de Mazatlán existen 744 
Integración de conocimiento en restaurantes mediante el análisis multicriterio para la toma

de decisiones

establecimientos de preparación y servicio de alimentos y de bebidas con categoría turística, mientras que la ocupación mediante llegada de turistas a establecimientos de hospedaje en 2016 fue de 2,154,570 (INEGI, 2017) observándose un crecimiento de $8.7 \%$ con respecto a 2015 (INEGI, 2016b). Este incremento en la actividad hotelera es ante todo en llegada de turistas nacionales provenientes del noreste del país, lo que se debe a la operación de la autopista a Durango desde hace tres años.

El artículo se estructura en integración de conocimiento y análisis multicriterio para toma de decisiones, en donde se abordan los aspectos teóricos; la metodología que aborda el MCDA a través del enfoque de ponderación aditiva simple (SAW por sus siglas en inglés); los resultados y, por último, las conclusiones.

\section{INTEGRACIÓN DE CONOCIMIENTO Y ANÁLISIS MULTICRITERIO PARA TOMA DE DECISIONES}

La fundamentación teórica de la investigación se presenta primeramente con la conceptualización de conocimiento e innovación $\mathrm{y}$, posteriormente se explican a partir de la gestión del conocimiento antecedentes de investigaciones. En un segundo momento se realiza el mismo esquema para el MCDA.

\subsection{Integración del conocimiento}

Davenport y Prusak (1998) señalan que el conocimiento es una mezcla en donde fluyen experiencias enmarcadas, valores e información voluntad, esto debido a la falta de conocimiento del entorno, de lo administrativo y en algunos casos financieros. En este sentido identificar los vínculos de conocimiento en la toma de decisiones a través del MCDA en la organización restaurantera que realiza actividades de innovación se considera pertinente, por dos motivos: en primer lugar, como una oportunidad de los gerentes y tomadores de decisiones para contar con herramientas eficaces en la identificación de las fuentes de conocimiento internas y externas que coadyuve con el ambiente de calidad y crear contextual y que, en las organizaciones, se inserta frecuentemente no sólo en documentos o repositorios, sino también en rutinas, procesos, prácticas y normas organizacionales. El conocimiento en la organización es un proceso que incorpora valores, actitudes y creencias y que permite conocer su entorno e intervenir dinámicamente en éste, con base en su experiencia (curva de aprendizaje) y sus habilidades (Fontalvo, Quejada \& Puello, 2011).

La innovación es la conversión de ideas y conocimientos en productos, procesos $\mathrm{o}$ servicios mejorados para el mercado, satisfaciendo así las necesidades de los ciudadanos, empresas y administraciones públicas (Afuah, 1999; Corma, 2011). Hjalager (2010) señala que la innovación de procesos en el turismo se refiere a las iniciativas que apuntan a la escalada de la eficiencia, la productividad y el flujo. La inversión en tecnología se convierte en la principal fuente, y generaría mayor eficiencia si se combina con otra estratégica y medidas de gestión, tales como el desarrollo de competencias y la gestión de recursos humanos. Un ejemplo en la empresa restaurantera de innovación de procesos es la rapidez en la preparación de alimentos.

La categoría de integración de conocimiento en la organización se enmarca en la gestión del conocimiento (GC) que en 
este estudio tiene el objetivo de apoyar la innovación y la generación de nuevas ideas en la organización. Por ello, la GC se puede abordar como una estrategia, un modelo interdisciplinar de los negocios o un proceso de competitividad y que conlleva a mejorar la capacidad competitiva del núcleo empresarial en la economía del conocimiento (Maheshwarkar y Sohani, 2016).

Abubakar, Elrehail, Alatailat y Elçi (2018) mencionan que los estudios de la GC han sido relacionados con resultados en la organización como el aprendizaje, la innovación, la calidad del producto, entre otros, ante ello proponen una visión integral del enfoque orientado a la integración del conocimiento y el desempeño organizacional que incorpore estilos de toma de decisiones intuitivos y racionales como moderadores entre la gestión del conocimiento y la actuación organizacional.

Nagles (2007) señala que, desde la perspectiva de la GC, la innovación necesita desarrollar tres acciones fundamentales: reconocer las oportunidades de innovación, identificar los conocimientos relevantes para aprovechar las oportunidades de innovación y generar soluciones sostenibles y rentables para la organización.

Desde la perspectiva de la organización inteligente se menciona el ciclo de la inteligencia con lo que demuestra su carácter dinámico. En este ciclo se manifiesta la percepción, la creación de conocimiento y la toma de decisiones que permite el aprendizaje y adaptación de la organización (Choo, 1999). El proceso del ciclo proviene de la cultura organizacional que permite a través de los significados compartidos de los miembros de la organización construir en lo individual y colectivamente nuevo conocimiento al convertir, compartir y sintetizar su conocimiento táctico y explícito. Posteriormente, sigue el eslabonamiento transversal del conocimiento de individuos, grupos e instituciones externos, lo cual coadyuva a estructurar la conducta ante una selección a través de premisas, reglas y rutinas con lo que, finalmente, la organización simplifica la toma de decisión, codifica $y$ transmite el saber pasado y proclama la competencia y la responsabilidad.

Por otra parte, la OCDE (2006) señala cuatro tipologías de innovación que pueden ser medidas, las de producto, de proceso, de marketing y organizacionales. Este organismo define a la innovación de procesos como la implementación de un método de producción o de operación nuevo o con mejoras significativas en las técnicas o equipo de software. La innovación de procesos busca la eficiencia en la organización. Entre las innovaciones de este tipo son: la gestión de la calidad total, la construcción de escenarios estratégicos y la gestión del conocimiento (Barbosa \& Dominique, 2012). De esta manera, la innovación del proceso de gestión del conocimiento se integra básicamente, por la generación, la codificación, la transferencia y la utilización del conocimiento (Wiig, 1997, citado por Cárcel \& Roldán, 2014).

\subsection{Análisis multicriterio para la toma de decisiones}

En la perspectiva teórica MCDA, la categoría de toma de decisiones se identifica en Simon (1997), como procesos cognitivos que se desarrollan en la mente del individuo y que tienen como meta primaria la elección de un curso de acción que ayude a resolver algún problema. Mientras que el análisis multicriterio es un campo avanzado de la investigación de operaciones, caracterizado 
Integración de conocimiento en restaurantes mediante el análisis multicriterio para la toma

de decisiones

principalmente por su orientación en el apoyo a la decisión. Al respecto Álvarez \& Leyva (2011), dicen que en el desarrollo de esta área de investigación han sobresalido dos formas de modelar las preferencias del decisor: el modelo funcional de la escuela norteamericana es la base de los enfoques de toma de decisiones multicriterio (MCDM, por sus siglas en inglés); y el modelo racional de la escuela europea, es la base del enfoque de ayuda a la decisión multicriterio (MCDA, por sus siglas en inglés). Para esta investigación se hace uso del análisis multicriterio como apoyo a la toma de decisiones, es decir, se utiliza el enfoque MCDA de la escuela europea.

El MCDA en décadas reciente se ha convertido en un apoyo relevante para la evolución teórica y práctica en la ciencia de la decisión. Además, en el desarrollo de los MCDA estos se han clasificado por categorías (Roy, 1985; Vincke, 1998; Zionts, 1992; y Pardalos, Siskos \& Zopounidis, 1995, citados por León \& Leyva, 2016) de acuerdo con lo siguiente:

a) La categoría que incluye a los métodos con un enfoque de síntesis y reducción a un solo criterio, sin aceptar incomparabilidades entre alternativas.

b) La categoría que resume todos los métodos con un enfoque de síntesis que conduce a una relación de sobreclasificación permitiendo la incomparabilidad entre alternativas, $y$

c) La categoría, que agrupa los métodos con un enfoque de discernimiento local interactivo, con interacciones de prueba y error.

En este sentido, Maheshwarkar y Sohani (2016) señalan que en el mundo de los negocios se ha acrecentado el reconocer al conocimiento como una fuerza impulsora de la organización, lo que precisa de una evaluación del desempeño de la GC en la organización, por lo que proponen los diferentes MCDA desde el proceso de jerarquía (AHP); la ponderación aditiva simple (SAW), la técnica para preferencia de pedido de similitud con la solución ideal (TOPSIS), y višekriterijumsko kompromisno rangiranje o compromiso clasificación (VIKOR), se utilizan para la evaluación de alternativas y el análisis de componentes principales (PCA), entre otros, todas las siglas son en inglés.

Por otra parte, la integración del conocimiento en la empresa restaurantera que proporciona servicios primordialmente al turismo se desenvuelve en un contexto para enfrentarse a los cambios bruscos de la economía debido al aumento de la incertidumbre operativa, así como a las distintas preferencias de los clientes y a ciclos de vida del producto-servicio más cortos. Zaei y Zaei (2014). En este sentido el enfoque teórico de la GC es una herramienta importante para las empresas de turismo que desean obtener ventajas competitivas a través de la hechura de mejores planes para el futuro, alcanzar un alto nivel de toma de decisiones y, en última instancia, aumentar la competitividad y obtener los máximos beneficios (Cooper, 2005, citado por Zaei \& Zaei (2014).

Por lo anterior, estudios que se han realizado con respecto a la GC y TD son escasos y con la utilización de diferentes metodologías, al respecto se tiene el caso de la investigación sobre la integración de conocimiento para la innovación en los restaurantes ubicados en Xalapa, México, Pérez y Alonso (2017) proponen un modelo de GC como estrategia de innovación, el cual contempla a la organización y con ello sus 
estrategias y personas; a la tecnología como facilitador y, por último a los procesos a través de los conocimientos. La propuesta se sustentó en información analizada proveniente de cuatro fuentes: las personales que los llevaron a indagar las experiencias, ideas, las opiniones, las intuiciones y las sugerencias; las empresariales, basadas en los procesos; la tecnología, internet, correo electrónico y buzón de voz; por último, el entorno, a través de la evaluación de los comensales, la información externa y el buzón de sugerencias.

La relación de los macroprocesos la GC y la toma de decisiones (TD) se estudian en empresas del sector del turismo, al demostrarse que el enriquecimiento sinérgico de la GC y la TD durante el ciclo de vida de las organizaciones contribuye al aprendizaje organizacional y a la eficacia de la toma de decisiones (Batista, Velázquez, Díaz \& Ronda, 2015). Sin embargo, estos autores, consideran insuficiente su institucionalización por lo que recurren a la armonización y alineación de los macroprocesos con el propósito de generar correlaciones positivas entre factores como las necesidades organizacionales, las manifestaciones del entorno y los recursos del sistema empresarial.

En este escenario, Batista et al. (2015) proponen el procedimiento para la exploración de la alineación de la TD con la GC aplicado en empresas relacionadas con el negocio del turismo en el destino turístico Holguín, Cuba, el cual se integra por tres fases: planificación, implementación, retroalimentación. La implementación se apoya en la información cualitativa en dos momentos: diagnosticar la alineación entre los objetivos de la TD y la GC en el que se revisa la existencia del plan de gestión de conocimiento, la disponibilidad de información y las características de las decisiones, entre otras; además analizar la idoneidad de la arquitectura del conocimiento para responder a la TD, que contempla las características de los sensores de información y del acceso a las fuentes, entre otras. La información cuantitativa se contempla en cálculo y análisis del coeficiente sintético de alineación de la gestión del conocimiento con la toma de decisiones, como Botti \& Peypoch (2013), León \& Leyva (2017), Leyva, Gastélum \& Urías (2016), Pulido \& Rodríguez (2016) \& Önder, Yıldırım \& Özdemir (2013). En tanto, Flores, Aceves, García y Peters (2015) evalúan el potencial del turismo.

León y Leyva (2017) utilizan la metodología multicriterio para el análisis comparativo de la competitividad de los destinos turísticos ubicados en el noroeste de México mediante el proceso metodológico que se realizó en dos etapas: la primera utiliza el método ELECTRE III para construir una relación valorada de superación, y la segunda usa un algoritmo evolutivo multiobjetivo para explotar esas relaciones y generar la clasificación de destinos; Önder, Yıldırım y Özdemir (2013) a través de los enfoques del Proceso de Jerarquía Analítica (AHP) y la técnica para preferencia de pedido de similitud con la solución ideal (TOPSIS) evalúan la competitividad de los destinos turísticos turcos. Los criterios son cuantitativos, tales como número de museos, número de hoteles, número de bahías o variables cualitativas, como la calidad de cocina, condiciones higiénicas, seguridad y protección, etc.

Flores, Aceves, García y Peters (2015) utilizaron el método de evaluación multicriterio (EMC) discreto proceso analítico jerárquico (PAJ), como una 
herramienta de ayuda a la toma de decisiones en la conservación algunas especies en la costa Chalacatepec, Jalisco. El propósito fue evaluar el impacto potencial del turismo sobre la anidación de tortugas en dos etapas: a) preparación y construcción y b) operación y mantenimiento, esto llevó a los autores a estimar el impacto de ocho amenazas turísticas: desmonte y despalme, excavaciones, rellenos, contaminación sonora, residuos sólidos, presencia de personal de obra y construcciones provisionales, iluminación artificial del frente de playa y concentración de visitantes.

\section{Metodología}

La exploración de metodologías de apoyo para la toma de decisiones en escenarios en los que intervienen múltiples variables o criterios de selección requiere del uso de técnicas que representen una herramienta fundamental para la investigación, debido a que ayuda a implementar métodos de análisis multidimensional, como es el problema que se desea tratar.

Para este trabajo, el ejercicio proviene de la encuesta realizada a ocho restaurantes ubicados en el puerto de Mazatlán, distribuidos espacialmente en la zona turística. Algunas razones que se consideraron en el estudio: ser pequeñas y medianas empresas; ubicarse en el malecón y zona dorada; contar con tres años como mínimo de funcionamiento; haber realizado actividades de innovación de procesos (introducción, desarrollo, ensayos y métodos de procesos, formación de capital humano e investigación y desarrollo interna) y, ser restaurantes que no se encuentran en hoteles. El estudio de caso se basó en un muestreo por área, el cual aplica a poblaciones o regiones que tienen características físicas, políticas o naturales similares, tal como lo indica Namakforoosh (2011) para la determinación de la muestra.

Adicionalmente, el instrumento para la recolección de datos se diseñó y adaptó con base en el manual de Oslo (OCDE, 2006), en la primera parte se preguntó sobre la información general de los establecimientos; número de empleados; porcentaje de usuarios nacionales e internacionales; la identificación del establecimiento por las particularidades de la cocina: nacional, internacional $u$ otros, así como si se realizan o no actividades innovadoras y sus tipos.

En la segunda parte del cuestionario, se encuentra la dimensión de vínculos integración de conocimiento y tecnología para la innovación de procesos, en ella se consideraron tres variables que contienen 41 ítems como alternativa de decisión, conforme la tabla 1.

TABLA 1. DIMENSIÓN DE INTEGRACIÓN DEL CONOCIMIENTO Y TECNOLOGÍA.

\begin{tabular}{lcccc}
\hline & \multicolumn{4}{c}{ Dimensión e ítems } \\
\cline { 2 - 5 } \multicolumn{1}{c}{ Variables } & $\begin{array}{c}\text { Información de } \\
\text { libre acceso }\end{array}$ & $\begin{array}{c}\text { Adquiere } \\
\text { (compra) }\end{array}$ & $\begin{array}{c}\text { Cooperación en } \\
\text { innovación }\end{array}$ & $\begin{array}{c}\text { Total ítems por } \\
\text { variables }\end{array}$ \\
\hline Internas & 3 & 1 & 1 & 5 \\
Fuentes comerciales y mercados exteriores & 5 & 5 & 5 & 15 \\
Fuentes que dependen del sector público & 3 & 4 & 4 & 11 \\
Fuentes de información general & 7 & 3 & 0 & 10 \\
Total ítems por dimensión & 18 & 13 & 10 & 41 \\
\hline
\end{tabular}

Fuente: elaboración propia basado en el manual de Oslo (OCDE, 2006). 
Para el caso particular, se opta por la utilización del enfoque de ponderación aditiva simple (SAW por sus siglas en inglés) propuesto por (Alireza, Majid, \& Rosnah 2010 p. 511), explicándose a través del proceso que se debe seguir para ejecutar este método multiatributo de la siguiente manera:

1. Elección de las alternativas. (León \& Leyva, 2017).

$\mathrm{A}=\{\mathrm{a} 1, \mathrm{a} 2, \ldots, \mathrm{aj}, \ldots, \mathrm{am}\}$,

donde $|A|=m$

\section{Identificación de criterios.}

3. Colección de los datos usando el cuestionario basado en la escala de valores de 0 y 1 .

4. Determinación de los pesos a través de la matriz de comparación (Alireza, et al, 2010, p. 511).

wj coeficiente de importancia relativa del criterio gj para $j=1,2, \ldots n$.

5. Cálculo de la matriz de decisión.

6. Cálculos y ordenamientos final.

$$
A_{i}=\sum W_{j} \cdot X_{i j} \quad \begin{gathered}
\mathrm{i}=1, \ldots 3, \\
\mathrm{j}=1, \ldots 8
\end{gathered}
$$

donde xij es el puntaje de la i-ésima alternativa con respecto al criterio j-ésimo, wj es el criterio ponderado (Alireza, et al, 2010, p. 512).

Esta metodología está diseñada para seleccionar y considerar los criterios de los restaurantes que fueron seleccionados para el estudio y con el cual se busca conocer qué organización es la que integra mejor el conocimiento y tecnología, haciendo uso de las fuentes de información de libre acceso, adquisición por compra y cooperación.

\section{ESTUDIO DE CASO COMO RESULTADO}

En este sentido se consideraron los vínculos que se requieren para incorporar conocimiento y tecnología en el desarrollo de innovaciones de procesos, entre estos se consideraron: 1) la información de libre acceso, 2) las se que adquieren (compra) y 3) las de cooperación activa en innovación (intercambio de conocimiento), señaladas en la tabla 1 , mismos que son agrupados en 3 criterios los cuales se muestran en la tabla 2 :

\subsection{Selección de alternativas}

En este tipo de estudios, Bartolini, Gallerani, Samoggia y Viaggi, (2005), consideran que, en una decisión, el conjunto de alternativas puede ser más o menos definida en algunos problemas de decisión y está abierto en el sentido de que estas pueden ser inventadas o descubiertas por el tomador de decisiones.

Es aquí donde las características básicas del análisis multicriterio representan el hecho de comparar alternativas sobre la base de una serie de criterios. De acuerdo con Roy (1991), las alternativas tienen que ser mutuamente excluyentes, consistentes en el tiempo y en el espacio y ser, además, comparables para cualquier característica diferente a partir de lo expresado por los criterios de evaluación.

De esta manera, se construye una matriz de alternativas $/ A$ /incorporándole su etiqueta de decisión (Tabla 3). En este caso, cada alternativa corresponde a un restaurante seleccionado, siendo $A=\left\{a_{1}, a_{2}, \ldots, a_{j}, \ldots, a_{m}\right\}$ el conjunto finito de alternativas, $\mid A /=m$ (León \& Leyva, 2017). 
Integración de conocimiento en restaurantes mediante el análisis multicriterio para la toma de decisiones

TABLA 2. DIMENSIÓN DE INTEGRACIÓN DEL CONOCIMIENTO Y TECNOLOGÍA.

\begin{tabular}{cc}
\hline Etiqueta & Criterios \\
\hline C1 & Información de libre acceso \\
C2 & Adquiere (compra) \\
C3 & Cooperación en innovación \\
\hline
\end{tabular}

Fuente: elaboración propia.

En el proceso de análisis, la recomendación puede tomar la forma de la selección de un subconjunto de alternativas en diferentes categorías o del ordenamiento global. En este caso, no se nombran los restaurantes, considerando que en el momento de la encuesta se señaló que sería anónima y para esta investigación no se utilizaría el nombre de estos.

\subsection{Recolección de los datos}

Usando el cuestionario como instrumento para la recolección de los datos, se les solicitó a los dueños, administradores y/o encargados de la operación del restaurante, que respondieran con 1 al responder «Si» y 0 al contestar «No» a las preguntas que se relacionaron con la integración del conocimiento y tecnología mediante el uso de información de libre acceso, la que se adquiere (compra) y de cooperación en innovación.
TABLA 3. ALTERNATIVAS DE RESTAURANTES Y SU ETIQUETA.

\begin{tabular}{cl}
\hline Alternativa & Etiqueta \\
\hline A1 & Restaurante 1 \\
A2 & Restaurante 2 \\
A3 & Restaurante 3 \\
A4 & Restaurante 4 \\
A5 & Restaurante 5 \\
A6 & Restaurante 6 \\
A7 & Restaurante 7 \\
A8 & Restaurante 8 \\
\hline
\end{tabular}

Fuente: elaboración propia.

\subsection{Determinación de los pesos}

Los pesos de los criterios a diferencia de otros métodos pueden ser considerados «coeficientes de importancia» o "valores de importancia relativa» $y$ no «tasas de sustitución» entre criterios, evitando con ello problemas de tipo compensatorio. En este estudio, el tomador de decisiones fue asistido para definir los pesos de cada uno de los criterios de acuerdo con la matriz de comparación (Alireza, et al, 2010). Donde, $w_{j}$ es el coeficiente de importancia relativa adjunta al criterio $g_{j}$ para $j=1,2, \ldots n$.

En este sentido, los datos se recogieron a partir de la opinión de cinco expertos en restaurantes con el cuestionario en que les fue proporcionado. Los pesos que se obtuvieron del consenso de los tomadores de decisiones se muestran en la Tabla 4.

TABLA 4. MATRIZ DE COMPARACIÓN PARA LA ASIGNACIÓN DE LOS VALORES RELATIVOS (PESOS).

\begin{tabular}{llllll}
\hline & $\begin{array}{l}\text { Información de libre } \\
\text { acceso }\end{array}$ & $\begin{array}{l}\text { Adquiere } \\
\text { (compra) }\end{array}$ & $\begin{array}{l}\text { Cooperación } \\
\text { innovación }\end{array}$ & en & Puma \\
\hline Información de libre acceso & 1.00 & 3.00 & 3.00 & 7.00 & 0.48 \\
Adquiere (compra) & 0.33 & 1.00 & 5.00 & 6.33 & 0.43 \\
Cooperación en innovación & 0.20 & 0.20 & 1.00 & 1.40 & 0.10 \\
TOTAL & & & & 14.73 & 1.00 \\
\hline
\end{tabular}

Fuente: elaboración propia.

\subsection{Cálculo de la matriz de desempeño.}

El modelo conceptual que se propone para analizar los vínculos para incorporar conocimiento y tecnología para el desarrollo de las innovaciones de procesos en los restaurantes de Mazatlán presenta una lista de atributos para evaluar la innovación. En total existe 41 factores a considerar y se representa en tres principales criterios. A continuación, los criterios para evaluar los restaurantes se muestran en la tabla 5 donde muestra los 
valores de cada alternativa (restaurante) con respecto a cada criterio, esta es la matriz de desempeño.

TABLA 5. MATRIZ PERFORMANCE DE LAS ALTERNATIVAS

\begin{tabular}{cccc}
\hline Alternativa/Criterios & $\mathrm{C}_{1}$ & $\mathrm{C}_{2}$ & $\mathrm{C}_{3}$ \\
\hline $\mathrm{A}_{1}$ & 4.75 & 1.29 & 0.29 \\
$\mathrm{~A}_{2}$ & 5.23 & 0.86 & 0.19 \\
$\mathrm{~A}_{3}$ & 4.75 & 1.72 & 0.10 \\
$\mathrm{~A}_{4}$ & 2.38 & 1.72 & 0.00 \\
$\mathrm{~A}_{5}$ & 3.33 & 1.72 & 0.48 \\
$\mathrm{~A}_{6}$ & 1.90 & 2.15 & 0.48 \\
$\mathrm{~A}_{7}$ & 3.80 & 1.29 & 0.67 \\
$\mathrm{~A}_{8}$ & 2.38 & 1.29 & 0.29 \\
\hline
\end{tabular}

Fuente: elaboración propia.

\subsection{Cálculos y ordenamientos finales.}

Con la información proporcionada, se construyó un modelo de integración de preferencias de las alternativas sintetizado como una relación de superación borrosa, para este propósito se utilizó modelo de suma aditiva ponderada (SAW) (Alireza, et al, 2010). La salida del resultante se muestra en la tabla 6, que presenta a medida que cada posición en el orden ha asignado un $W_{i}$ peso, se calcula la suma ponderada.

TABLA 6: RANKING GLOBAL DE ALTERNATIVAS

\begin{tabular}{ccc}
\hline Posición & Código & Resultado \\
\hline 1 & $\mathrm{~A}_{3}$ & 6.57 \\
2 & $\mathrm{~A}_{2}$ & 6.47 \\
3 & $\mathrm{~A}_{1}$ & 6.23 \\
4 & $\mathrm{~A}_{7}$ & 5.76 \\
5 & $\mathrm{~A}_{5}$ & 5.52 \\
6 & $\mathrm{~A}_{6}$ & 4.52 \\
7 & $\mathrm{~A}_{4}$ & 4.10 \\
8 & $\mathrm{~A}_{8}$ & 3.95 \\
\hline
\end{tabular}

Fuente: elaboración propia.

Los resultados sugieren la clasificación final del ordenamiento, en la que destacan los restaurantes A3, A2 y A1, como los que más y mejor han logrado la integración de conocimiento y tecnología, le siguen los restaurantes A7 y A5 que resultaron con el mismo rango de 5, los establecimientos $\mathrm{A6}$, A4 y A8 de los datos obtenidos sugieren estar por debajo, debido a que estas empresas incorporan bajos elementos de conocimiento para innovación.

Si bien estos restaurantes han innovado en procesos se observa que sus alternativas de decisión para incorporar conocimiento no es aprovechado lo suficientemente, esto se relaciona con la perspectiva de que la innovación en el destino Mazatlán "parte de un esfuerzo individual y no de una actividad vinculada" (Larrañaga \& León, 2017, p. 30), por lo que la utilización del MCDA aumentaría la retroalimentación interna y externa de conocimiento efectivo en este sector lo que acrecentaría la calidad del servicio y sus ventajas competitivas.

Adicionalmente, tomando en consideración el número de variables de cada criterio, se realizaron los cálculos de manera individual para analizar la integración de conocimiento y tecnología al establecimiento: 1) por uso de información de libre acceso, 2) la que se adquiere (compra) y 3) por cooperación en innovación. Considerando que para cada criterio existen diferentes pesos, los resultados se muestran en la tabla 7.

Los resultados obtenidos en la integración de conocimiento que realizan los restaurantes, la alternativa A5 se ubica en la primera posición como la incorpora procesos la información de libre acceso seguida por A8. Por su parte, el establecimiento que mayormente adquiere este proceso es la alternativa A7 y le secunda la A6. Finalmente, quien lo quien lo hacer por cooperación en innovación correspondió a la alternativa A5 y la A2.

El destino Mazatlán, en el tiempo se ha considerado como tradicional, sin embargo, los resultados individuales demuestran que 
los tomadores decisiones han incorporado conocimientos del contexto interno y externo como una necesidad de las pequeñas y medianas empresas, para seguir compitiendo en un mercado en crecimiento.

TABLA 7: RANKING INDIVIDUAL DE ALTERNATIVAS SEGÚN INTEGRACIÓN DE CONOCIMIENTO Y TECNOLOGÍA

\begin{tabular}{cccc}
\hline $\begin{array}{c}\text { Posic } \\
\text { ión }\end{array}$ & $\begin{array}{c}\text { Información de } \\
\text { libre acceso }\end{array}$ & $\begin{array}{c}\text { Adquiere } \\
\text { (compra) }\end{array}$ & $\begin{array}{c}\text { Cooperación en } \\
\text { innovación }\end{array}$ \\
\hline 1 & $\mathrm{~A}_{5}$ & $\mathrm{~A}_{7}$ & $\mathrm{~A}_{5}$ \\
2 & $\mathrm{~A}_{8}$ & $\mathrm{~A}_{6}$ & $\mathrm{~A}_{2}$ \\
3 & $\mathrm{~A}_{7}$ & $\mathrm{~A}_{2}$ & $\mathrm{~A}_{8}$ \\
4 & $\mathrm{~A}_{2}$ & $\mathrm{~A}_{3}$ & $\mathrm{~A}_{1}$ \\
5 & $\mathrm{~A}_{3}$ & $\mathrm{~A}_{4}$ & $\mathrm{~A}_{3}$ \\
6 & $\mathrm{~A}_{4}$ & $\mathrm{~A}_{1}$ & $\mathrm{~A}_{7}$ \\
7 & $\mathrm{~A}_{6}$ & $\mathrm{~A}_{8}$ & $\mathrm{~A}_{6}$ \\
8 & $\mathrm{~A}_{1}$ & $\mathrm{~A}_{5}$ & $\mathrm{~A}_{4}$ \\
\hline
\end{tabular}

Fuente: elaboración propia.

Al respecto Davenport y Prusak (1998), mencionan la relevancia de la valoración de este recurso intangible para este tipo de empresas, de ahí que el MCDA apoye a la toma de decisiones en un contexto real.

\section{CONCLUSIONES}

El entorno globalizador y competitivo, así como el desarrollo de la economía del conocimiento en el que se encuentran las empresas restauranteras las llevan a realizar actividades de innovación que se manifiestan en actualización y formación de capital humano y con ello a introducir y desarrollar nuevos métodos en los procesos, de esta manera se aprovecha el conocimiento del recurso humano $y$ de repositorios organizacionales, de los competidores, colaboradores, proveedores, entre otros.

Además, estos establecimientos que proporcionan servicios a visitantes cada vez más exigentes e informados, retroalimentan a la organización mediante quejas o comentarios no solo en la calidad del servicio, sino en los procesos de elaboración de los alimentos, en la atención, del mantenimiento de la infraestructura e integración de tecnologías, entre otros. La innovación de procesos en la organización restaurantera es una ventaja competitiva ya que es difícil de imitar, en comparación con la innovación de productos (Weiermair, 2004), por lo que el estudio de la selección de los atributos para la integración de conocimiento en la innovación de procesos a través del método SAW proporciona información de la toma de decisiones eficaz

El beneficio de la identificación de la alternativa de decisión para la integración de conocimiento a través de un método multiatributo, en la innovación de proceso en la empresa restaurantera ubicada en el destino turístico de Mazatlán, Sinaloa, México, no solo apoya a los gerentes y operadores de los establecimientos, sino que contribuye en las gestiones que realizan las entidades públicas y privadas, en este caso las Secretarías de Turismo nacional y local, así como la Cámara Nacional de la Industria Restaurantera, para orientar al sector y, en su caso, a los agremiados en la utilización de herramientas que faciliten la toma de decisiones de manera más objetivas y generar estrategias encaminadas a lograr ventajas competitivas en la industria que se observa en crecimiento en este destino.

Por lo que respecta a la revisión del estado del arte, los estudios de MCDA con experiencias similares en el turismo se tienen como autores a Botti \& Peypoch (2013), León \& Leyva (2017), Leyva, Gastélum \& Urías (2016), Pulido \& Rodríguez (2016) y Önder, Yıldırım \& Özdemir (2013), lo que permitió descubrir que las aplicaciones para el análisis multicriterio, y en particular SAW, se encuentran en numerosas investigaciones, derivadas de que la mayoría son los problemas 
de la vida real, los cuales se encuentran entre subjetividad y objetividad, en donde lo que importa es la opinión de los involucrados requiriendo que se formulen criterios tangibles y prioridades de decisión.

Sin embargo, con relación a las aplicaciones para estudiar la integración de conocimiento y tecnología en los restaurantes, son pocos los que han dado registro de ello. Además, no se ha planteado una guía clara del proceso para su determinación, así como las dificultades vividas en el mismo camino. Por lo tanto, fue posible establecer un ordenamiento de estos establecimientos, en disposición ascendente, permitiendo evaluar de manera integral esta investigación, de tal manera que destacan los restaurantes A3, A2 y A1, como los que más y mejor han logrado este proceso.

Por último, las empresas turísticas y entre ellas las restauranteras se desenvuelven en un contexto de cambios bruscos de economía debido al ciclo de vida del producto servicio más corto (Zaei \& Zaei, 2014), por lo que se recomienda para trabajos futuros la utilización de este procedimiento de unir la GC y MCDA para analizar la integración de conocimiento y tecnología para la innovación lo que contribuiría, además, con la hechura de mejores planes y alcanzar un alto nivel de toma de decisiones.

\section{REFERENCES}

Abubakar, A.M., Elrehail, H., Alatailat, M.A. \& Elçi, A. (2018). Knowledge management, decision-making style and organizational performance. Journal of Innovation \& Knowledge, JIK-54, 1-11. https://doi.org/10.1016/j.jik.2017.07.003

Afuah, A. (1999). La dinámica de la innovación organizacional, el nuevo concepto para lograr ventajas competitivas y rentabilidad. México: Oxford.

Alireza, A., Majid M., \& Rosnah M. (2010). Simple additive weighting approach to personnel selection problem.
International Journal of Innovation, Management and Technology, 1(5), 511-515.

Álvarez, P., \& Leyva, J.C. (2011). Sistemas de apoyo a la decisión multicriterio en grupo: los modos de coordinación paralelo y secuencial del proceso de decisión. En E., Avilés Ochoa, M. Rodríguez Peñuelas, Marcos teóricos para el estudio de las ciencias económicoadministrativas (1st. ed., 47-77). México: Juan Pablos.

Aparecido, C. \& Marques, J.L. (2016). Innovatividad, creatividad y gestión del conocimiento en bares, restaurantes y clubes nocturnos: un estudio en los establecimientos de Curitiba - PR. Revista Brasileira de Pesquisa em Turismo. $10 \quad$ (1), 18-43. http://dx.doi.org/10.7784/rbtur.v10i1.1013

Barbosa, B. \& Dominique, S. (2012). La innovación de los procesos. Diferenciación en los servicios turísticos. Estudios y Perspectivas en Turismo. 21(4), 963 - 976.

Bartolini, F. Gallerani, V. Samoggia, A. \& Viaggi, D. (2005). «Methodology for multicriteria analysis of agrienvironmental schemes», Technical Report, Sixth Framework Programme. University of Bologna.

Batista, C., Velázquez, R, Díaz, C. \& Ronda, G. (2015). Alineación entre toma de decisiones y gestión del conocimiento. El caso de las empresas relacionadas con el negocio del turismo. Ingeniare. Revista Chilena de Ingeniería. 23 (4), 540-555.

Botti, L. \& Peypoch, N. (2013). Multi-Criteria ELECTRE method and destination competitiveness. Tourism Management Perspectives, 6, 108-113.

Cárcel, F. \& Roldán, C. (2014). Principios básicos de la gestión del conocimiento y su aplicación a la empresa industrial en sus actividades tácticas de mantenimiento y explotación operativa: Un estudio cualitativo. Intangible Capital. 9(1), 91-125. http://dx.doi.org/10.3926/ic.341

Corma, F. (2011). Innovación, innovadores y empresa innovadora. España: Editorial Díaz de Santos.

Choo, Ch. (1999). La organización inteligente. El empleo de la información para dar significado, crear conocimiento y tomar decisiones. México: Oxford University Press.

Davenport, T. \& Prusak, L. (1998). Working knowledge: how organizations manage what they know. Boston. Harvard Business School Press

Flores, Y. Aceves, F. García, A. \& Peters, E.M. (2015). Análisis multicriterio del impacto potencial del turismo en la anidación de las tortugas marinas en Chalacatepec, Jalisco. Nova Scientia, 7 (14), 644-673.

Flores, S. \& Leyva, J.C. (2015). Análisis multicriterio para la toma de decisiones en el sector turístico: un acercamiento teórico-conceptual. En M. Velarde, M.A. Santillán y M.R. Contreras (Coords.). Perspectivas y tendencias del turismo en México (65-84). México: Juan Pablos Editor. 
Integración de conocimiento en restaurantes mediante el análisis multicriterio para la toma

de decisiones

Fontalvo, T., Quejada, R. \& Puello, J. (2011). La gestión del conocimiento y los procesos de mejoramiento. Revista Dimensión Empresarial, 9(1), 80-87.

Franch, K., Herrera, K. \& Losada, A. (2013). La gestión del conocimiento como herramienta de apoyo al proceso de decisiones. RIPS Revista de Investigaciones Políticas y Sociológicas, 12(1), 115-156.

Fraj, E., Matute, J. \& Melero, I. (2015). Environmental strategies and organizational competitiveness in the hotel industry: The role of learning and innovation as determinants of environmental success. Tourism Management, $\quad 46, \quad 30-42$. http://dx.doi.org/10.1016/j.tourman.2014.05.009

Hjalager, A. M. (2010), A review of innovation research in tourism. Tourism Management, 31(2010), 1-12.

Hernández, V. (2016). La gestión del conocimiento en las organizaciones. Buenos Aires. Alfaomega Grupo Editor.

INEGI (2016a). Censos económicos 2014. La industria restaurantera en México.

INEGI (2016b). Anuario estadístico y geográfico 2016.

INEGI (2017). Anuario estadístico y geográfico 2017.

Larrañaga, A.M. \& León, M. (2017). Elementos de innovación en hoteles. Mazatlán, Sinaloa, México. $3 C$ Empresas: Investigación y Pensamiento Crítico, 6 (2), 20-32. http://dx.doi.org/10.17993/3cemp.2017.060230.20-32

Lee, C., Rob Hallak, R. \& Sardeshmukh, S. (2016). Innovation, entrepreneurship, and restaurant performance: A higherorder structural model. Tourism Managment, 53, 215-228. http://dx.doi.org/10.1016/j.tourman.2015.09.017

León \& Leyva (2016). Metodología multicriterio para el análisis de la competitividad de los destinos turísticos. El caso del noroeste de México. México: Ediciones del lirio.

León, M. \& Leyva, J. C. (2017). A multicriteria decision aid for evaluating the competitiveness of tourist destinations in the Northwest of Mexico. Turismo y Sociedad, XXI, 51-67. https://doi.org/10.18601/01207555.n21.03

Leyva, J.C., Avilés, E., \& Zepeda, J.J. (2009). Prólogo. En Herramientas operativas para el análisis multicriterio del desarrollo económico local. México: Plaza y Valdés.

Leyva López, J. C., Gastélum Chavira, D. A. \& Urías Ruiz, M. (2016). A comparative approach of economic sectors in Sinaloa, Mexico, based on multicriteria decision aiding. EconoQuantum, 13(1), 97-124.

Maheshwarkar, M. \& Sohani, N. (2016). Evaluation of knowledge management levels based on multi criteria analysis. Management Science and Engineering. 10 (2), 523.

Mardani, A., Jusoh, A., MD Nor, K., Khalifah, Z., Zakwan, N. \& Valipour, A. (2015). Multiple criteria decision-making techniques and their applications - a review of the literature from 2000 to 2014. Economic ResearchEkonomska Istraživanja. 28 (1), 516-571. https://doi.org/10.1080/1331677X.2015.1075139

Medina D., Medina, A. \& Nogueira, D. (2017). Procesos y factores calves en la gestión del conocimiento. Universidad y Sociedad, 9(2), 16-23.

Namakfroroosh, M. N. (2011). Metodología de la investigación. Ed. 2. Limusa. México.

Nagles G. (2007). La gestión del conocimiento como fuente de innovación. Revista Escuela de Administración de Negocios, 61, 77-87.

OCDE (2006). Manual de Oslo. España: Grupo Tragsa.

Önder, E., Yıldırım, B. \& Özdemir, M. (2013). Multi Criteria Decision Making Approach for Evaluating Tourism Destinations in Turkey. Academic Journal of Tourism and Management Researches, 1, 1-15.

Pérez, E.J. \& Alonso, J. (2017). La gestión del conocimiento como estrategia de innovación en la industria restaurantera de Xalapa, Veracruz. Horizontes de la Contaduría en las Ciencias Sociales. 3 (6), 61-68.

Pulido, J. I. \& Rodríguez, B. (2016). Reinterpreting the World Economic Forum's global tourism competitiveness index. Tourism Management Perspectives, 20, 131-140.

Rodríguez, M.C. (2004). Racionalidad y toma de decisiones en las organizaciones. En L.M. Hirose, S. Alvarado, A. Barba y R. Soria (Coord.). Los estudios organizacionales en México (pp. 117-140). México: Plaza y Valdez

Roy, B. (1991). "The outranking approach and the foundations of electre methods". Theory and decision, 31(1), 49-73.

Salas de la Rosa, N., \& Garza Ruíz, M. (2016). Transferencia de conocimiento: un mecanismo de competitividad para las PYMES en el Estado de Nuevo León. Inquietud Empresarial, 16(1), 147-173.

Simon, H. A. (1997). Administrative behavior: a study of decision-making processes in administrative organizations. United State of America: The free press.

Weiermair, K. (2004), Product improvement or innovation: what is the key to success in tourism? En OECD innovation and growth in tourism: Conference Papers. http://www.oecd.org/cfe/tourism/34267947.pdf

Zaei, M.E. \& Zaei, M.E. 2014. Knowledge management in hospitality and tourism industry: a $\mathrm{km}$ research perspective. Information and Knowledge Management. 4 (9), 114-122. 